\title{
RESULTS OF THE 2007 INTERNATIONAL BUTTERFLY COUNTS IN SASKATCHEWAN
}

MIKE GOLLOP, 51 Welker Crescent, Saskatoon, SK S7H $3 M 3$ and ANNA LEIGHTON, 328 Saskatchewan Crescent West, Saskatoon, SK S7M 0A4

Nine International Butterfly Counts (1JC) were conducted in Saskatchewan in 2007, one more than in 2006. ${ }^{2}$ Counts in 2007 were conducted at Bjorkdale, Duck Mountain Provincial Park, Fort Qu'Appelle, Last Mountain Lake National Wildlife Area, Nisbet Forest, Pasquia Hills, Preeceville, Regina and Saskatoon. Duck Mountain and Nisbet were not done in 2006 but had been done in previous years, while the Last Mountain Lake count was new. Counts at Eastend and Waskesiu River were done in 2006 but not in 2007. Count statistics are presented in Table 1 , and count results in Table 2.

Sixty species were recorded in 2007 , compared to 62 in 2006 and 57 in $2005 .^{1,2}$ The total number of butterflies counted was 4007 , compared with 3669 in 2006 and 6972 in 2005 (of which 5389 were Painted Ladies). Butterflies per party-hour is used as a measure to relate butterfly numbers to observer effort to provide a comparable index of abundance over years. Butterflies per party-hour was 48 for the nine counts in 2007, the same as for the eight counts in 2006.

Count conditions in 2007 were consistent with those in previous years, ranging from a low temperature of $16^{\circ}$ at Regina to a high of $31^{\circ}$ at Preeceville. Wind speeds exceeding $30 \mathrm{~km} / \mathrm{hr}$ at Duck Mountain, Fort Qu'Appelle and Preeceville may have had some influence on the number of open field butterflies that were seen.

Species totals for each count ranged from 16 at Duck Mountain to 36 at Saskatoon and averaged 24 for the nine counts. This was up from 20 in 2006 and 18 in 2005. Twenty or more species is very respectable for a single-day butterfly count, and over 30 is exceptional. All but two counts exceeded 20 species in 2007 as species diversity improved with the generally adequate levels of moisture and warm mid-summer temperatures.

Numbers of individuals ranged from 153 at Last Mountain Lake to 1013 at Bjorkdale. The number of individuals per party-hour ranged from 22 at Last Mountain Lake to 127 at Bjorkdale compared with a range of 19 at Fort Qu'Appelle to 164 at Eastend in 2006.

The number of observers ranged from one at Duck Mountain, Bjorkdale and Pasquia Hills to 13 at Saskatoon and 10 at Fort Qu'Appelle. A total of 96 party-hours was logged, ranging from seven at Duck Mountain, Ft. Qu'Appelle, Preeceville and Last Mountain Lake to 32.5 at Saskatoon.

The highest combined count of an individual species was 1015 Clouded Sulphurs. Unusually high species counts at individual locations were 115 Canadian Tiger Swallowtails at Duck 


\begin{tabular}{|c|c|c|c|c|c|c|c|c|c|c|}
\hline 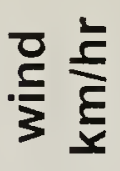 & $\begin{array}{l}\tilde{N} \\
\tilde{1} \\
\underline{\sigma}\end{array}$ & ㅇ & $\begin{array}{c}\stackrel{\infty}{+} \\
\stackrel{\sim}{\sim}\end{array}$ & 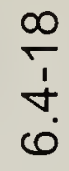 & 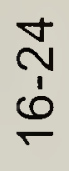 & $\frac{n}{5}$ & $\begin{array}{l}\stackrel{O}{+} \\
\stackrel{1}{+} \\
\stackrel{\sim}{v}\end{array}$ & $\frac{10}{0}$ & $\frac{9}{\frac{9}{5}}$ & $\underset{\substack{\infty \\
⿱ \\
0}}{ }$ \\
\hline 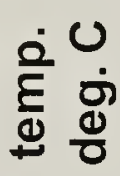 & 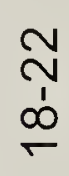 & $\begin{array}{l}\stackrel{\infty}{N} \\
\underline{1} \\
\underline{2}\end{array}$ & 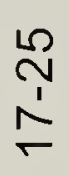 & 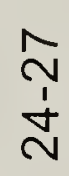 & $\frac{\stackrel{+}{\sim}}{\stackrel{\sim}{N}}$ & $\frac{\infty}{\stackrel{\infty}{\sim}}$ & $\begin{array}{l}\grave{n} \\
\stackrel{d}{d}\end{array}$ & $\begin{array}{l}\stackrel{\infty}{\sim} \\
\stackrel{1}{N}\end{array}$ & $\begin{array}{l}\stackrel{0}{N} \\
\stackrel{1}{\sigma}\end{array}$ & $\begin{array}{l}\tilde{m} \\
\dot{\omega}\end{array}$ \\
\hline $\begin{array}{c}\sum_{0} \\
\text { के } \\
\text { o̊ }\end{array}$ & $\frac{8}{\frac{8}{1}}$ & চ & $\frac{8}{0}$ & $\stackrel{8}{\circ}$ & $\frac{8}{0}$ & ڤ̊ & $\frac{10}{\frac{1}{10}}$ & $\stackrel{L}{N}$ & $\begin{array}{l}\infty \\
\infty\end{array}$ & \\
\hline 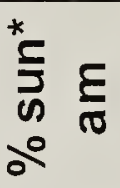 & $\stackrel{ㅇ}{\circ}$ & ণ & $\frac{n}{\frac{1}{1}}$ & 웅 & $\frac{8}{\circ}$ & 음 & $\begin{array}{l}0 \\
\stackrel{1}{1} \\
\stackrel{1}{ }\end{array}$ & ৪ & $\stackrel{\circ}{\circ}$ & \\
\hline$\stackrel{\frac{O}{c}}{\frac{c}{0}}$ & $\begin{array}{l}8 \\
0 \\
0 \\
5 \\
0 \\
0 \\
\infty \\
0\end{array}$ & $\begin{array}{l}\frac{L}{L} \\
\frac{1}{2} \\
\\
\vdots \\
m \\
0 \\
0 \\
0\end{array}$ & $\begin{array}{l}8 \\
8 \\
\infty \\
1 \\
8 \\
8 \\
0\end{array}$ & 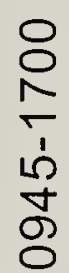 & $\begin{array}{c}0 \\
\text { N} \\
\frac{1}{1} \\
0 \\
\text { m } \\
0\end{array}$ & $\begin{array}{l}8 \\
\frac{8}{2} \\
\frac{1}{0} \\
8 \\
8 \\
0\end{array}$ & $\begin{array}{l}8 \\
8 \\
0 \\
\\
0 \\
8 \\
8 \\
0\end{array}$ & $\begin{array}{l}0 \\
0 \\
\frac{8}{\sigma} \\
1 \\
0 \\
m \\
0 \\
0\end{array}$ & 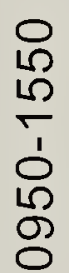 & \\
\hline 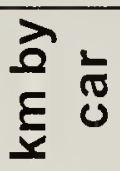 & 0 & 0 & & 0 & & $m$ & 0 & 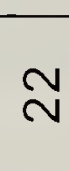 & 0 & \\
\hline $\begin{array}{l}\frac{5}{0} \\
\varepsilon \\
\underline{x}\end{array}$ & $\infty$ & $\begin{array}{l}\sim \\
\stackrel{\sim}{\sim}\end{array}$ & $\stackrel{\bullet}{\sigma}$ & $\underset{\bullet}{\bullet}$ & $\stackrel{\nabla}{\sim}$ & ما & $\underset{0}{\forall}$ & $N$ & $\sigma$ & $\begin{array}{l}\Omega \\
\infty\end{array}$ \\
\hline $\begin{array}{l}\text { 둥 } \\
\text { 둥 }\end{array}$ & & $\begin{array}{l}0 \\
\stackrel{0}{0} \\
\stackrel{0}{0}\end{array}$ & & $\Lambda$ & & $m$ & $\Lambda$ & $r$ & $\infty$ & \\
\hline $\begin{array}{l}\text { 눙 } \\
\stackrel{0}{\circ}\end{array}$ & $\Lambda$ & $\stackrel{م}{\stackrel{0}{0}}$ & $\lambda$ & $\Lambda$ & $\stackrel{\text { ஸn }}{\underset{m}{ }}$ & $\infty$ & $\lambda$ & م & $\infty$ & চ) \\
\hline $\begin{array}{l}\text { to } \\
0 \\
0\end{array}$ & $r$ & $\sigma$ & $\stackrel{\circ}{\leftarrow}$ & $m$ & $\stackrel{m}{\leftarrow}$ & $r$ & $\nabla$ & $r$ & $m$ & $\stackrel{\infty}{m}$ \\
\hline 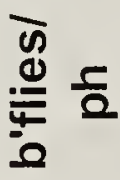 & $\stackrel{\curvearrowright}{\sim}$ & $\hat{\sim}$ & $\stackrel{\sim}{N}$ & 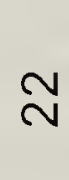 & $\stackrel{\sim}{N}$ & $\underset{\sim}{\sim}$ & Бо & ১্ & $\underset{\forall}{\stackrel{\forall}{ }}$ & $\stackrel{\infty}{+}$ \\
\hline 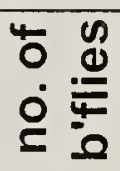 & ঠ & 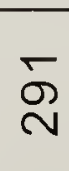 & ڤొ & $\stackrel{m}{2}$ & 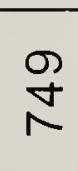 & $\frac{m}{0}$ & $\begin{array}{l}\text { } \\
\stackrel{\mathbb{N}}{\infty}\end{array}$ & $\frac{N}{\pi}$ & $\begin{array}{l}\text { } \\
10 \\
\text { N }\end{array}$ & $\begin{array}{l}1 \\
8 \\
8\end{array}$ \\
\hline $\begin{array}{l}\text { to } \\
\stackrel{0}{\circ} \\
\stackrel{0}{n}\end{array}$ & $\stackrel{\bullet}{\leftarrow}$ & $\stackrel{ \pm}{\sim}$ & $\overleftarrow{\sim}$ & $\approx$ & $\stackrel{\text { }}{\circ}$ & $\stackrel{\bullet}{\sim}$ & $\overleftarrow{\sim}$ & $\stackrel{m}{m}$ & $\stackrel{\infty}{\leftarrow}$ & 8 \\
\hline 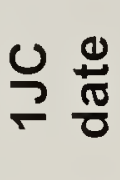 & z & $\frac{z}{\bar{N}}$ & $\stackrel{Z}{\stackrel{Z}{\sim}}$ & & 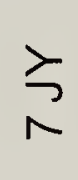 & 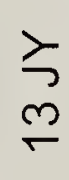 & $\underset{⿱}{\check{Z}}$ & う & 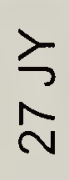 & $\stackrel{3}{5}$ \\
\hline$\stackrel{\dot{0}}{\lambda} \frac{0}{\square}$ & $\sim$ & $\underset{\sim}{\sim}$ & $\stackrel{\sim}{\sim}$ & $\tau$ & $m$ & $\Lambda$ & $\bullet$ & $\Lambda$ & $\sim$ & \\
\hline 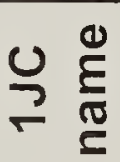 & $\sum_{0}^{\circ}$ & $\begin{array}{c}\text { o } \\
\mathbb{1} \\
\alpha\end{array}$ & 胥 & $\sum_{\lrcorner}$ & $\frac{O}{\ddot{\Lambda}}$ & $\frac{\circ}{\infty}$ & $\frac{0}{2}$ & $\begin{array}{l}\frac{T}{\pi} \\
0\end{array}$ & $\frac{\bigsqcup}{\bar{Z}}$ & $\frac{\omega}{\frac{\omega}{0}}$ \\
\hline
\end{tabular}

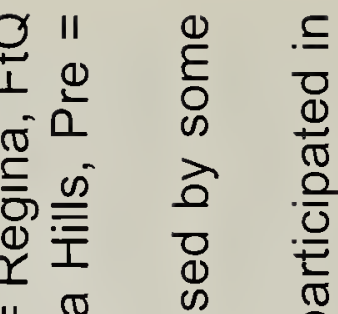

$11 \frac{1}{2} \frac{2}{2}$

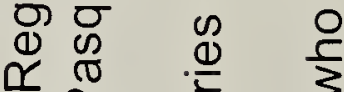

ㅁํㅇำ

त I $\frac{\Phi}{\sigma}$ ठ

ว능

늠 음

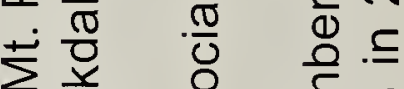

응 की है ब

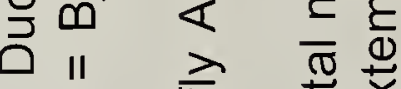

II 으 音 항

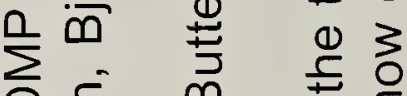

ธิ

๗유 तथ

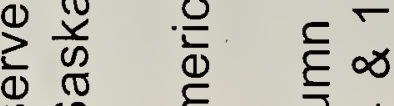

ஸा है

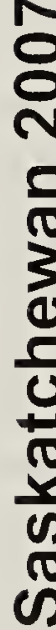

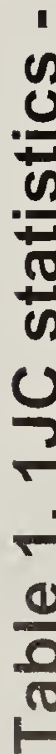

웡

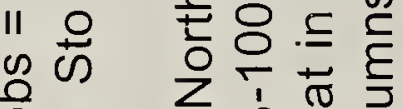
तั

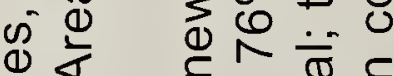

(1) ब유

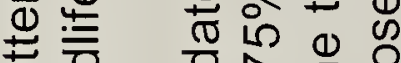

을 물도

11 耏完

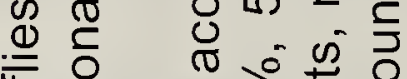

。

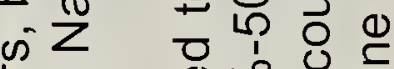

产 ब 음 党

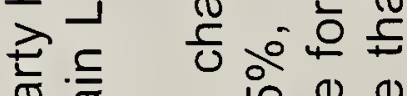

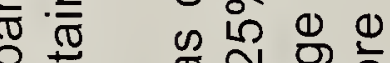
II 들을 काष ब ब 4 요 ॥ $\| \vec{\Sigma}$ की 닌용 किष is $\overline{\overline{0}}$

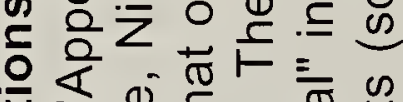

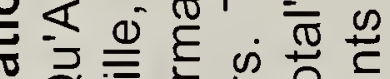
$>0>0$

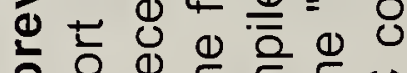

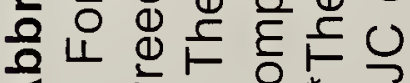
争 


\section{Table 2. 1JC results- Saskatchewan 2007}

\begin{tabular}{|c|c|c|c|c|c|c|c|c|c|c|}
\hline SPECIES NAME & $\begin{array}{l}\text { DMP } \\
9 \mathrm{JN}\end{array}$ & $\begin{array}{c}\operatorname{Reg} \\
21 \mathrm{JN}\end{array}$ & $\begin{array}{c}F t Q \\
23 \mathrm{JN}\end{array}$ & $\begin{array}{l}\mathrm{LML} \\
5 \mathrm{JY}\end{array}$ & $\begin{array}{l}\text { Sto } \\
7 \mathrm{JY}\end{array}$ & $\begin{array}{c}\text { Bjo } \\
13 \mathrm{JY}\end{array}$ & $\begin{array}{c}\text { Pre } \\
14 \mathrm{JY}\end{array}$ & $\begin{array}{c}\mathrm{PaH} \\
18 \mathrm{JY}\end{array}$ & $\begin{array}{c}\mathrm{NiF} \\
27 \mathrm{JY}\end{array}$ & totals \\
\hline $\begin{array}{l}\text { Silver-spotted Skipper } \\
\text { Northern Cloudywing } \\
\text { Dreamy Duskywing }\end{array}$ & $\begin{array}{l}1 \\
4 \\
\end{array}$ & 5 & & & 3 & & & 1 & & $\begin{array}{l}8 \\
2 \\
4\end{array}$ \\
\hline \multicolumn{11}{|l|}{$\begin{array}{l}\text { Juvenal's Duskywing } \\
\text { Afranius Duskywing } \\
\text { Persius Duskywing }\end{array}$} \\
\hline $\begin{array}{l}\text { Grizzled Skipper } \\
\text { C. Checkered Skipper } \\
\text { Common Sootywing }\end{array}$ & & & 1 & & 1 & & & & & 2 \\
\hline $\begin{array}{l}\text { Unident. Skipper } \\
\text { Arctic Skipper } \\
\text { Least Skipper }\end{array}$ & 8 & & 2 & 10 & 1 & & & & 1 & $\begin{array}{l}12 \\
10\end{array}$ \\
\hline $\begin{array}{l}\text { Garita Skipperling } \\
\text { European Skipper } \\
\text { Uncas Skipper }\end{array}$ & & 10 & 27 & 2 & 5 & & 11 & & & 55 \\
\hline \multicolumn{11}{|l|}{$\begin{array}{l}\text { C. Branded Skipper } \\
\text { Plains Skipper } \\
\text { Dakota Skipper }\end{array}$} \\
\hline $\begin{array}{l}\text { Nevada Skipper } \\
\text { Peck's Skipper } \\
\text { Draco Skipper }\end{array}$ & & & & & 4 & & & 2 & & 6 \\
\hline $\begin{array}{l}\text { Tawny-edged Skipper } \\
\text { Long Dash Skipper } \\
\text { Rhesus Skipper }\end{array}$ & & 7 & 4 & $\begin{array}{l}6 \\
1\end{array}$ & 4 & 5 & & 12 & & $\begin{array}{l}6 \\
33\end{array}$ \\
\hline $\begin{array}{l}\text { Delaware Skipper } \\
\text { Woodland Skipper } \\
\text { Hobomok Skipper }\end{array}$ & 4 & 10 & 1 & & & & & & & 15 \\
\hline $\begin{array}{l}\text { Dun Skipper } \\
\text { Dusted Skipper } \\
\text { Oslar's Roadside Skipper }\end{array}$ & & & & & 2 & 12 & 1 & 7 & 1 & 23 \\
\hline $\begin{array}{l}\text { Com. Roadside Skipper } \\
\text { Kahli Swallowtail } \\
\text { Old World Swtail (Dods) }\end{array}$ & & & 1 & 1 & 1 & & & & & $\begin{array}{l}2 \\
1\end{array}$ \\
\hline $\begin{array}{l}\text { Old World Sw'tail (Huds) } \\
\text { Anise Swallowtail } \\
\text { Anise/Old World Sw'tail }\end{array}$ & & & & & & & & 3 & & 3 \\
\hline $\begin{array}{l}\text { Can. Tiger Swallowtail } \\
\text { Unident. White } \\
\text { Western White }\end{array}$ & 115 & $\begin{array}{l}17 \\
1\end{array}$ & 9 & 7 & $\begin{array}{l}3 \\
6\end{array}$ & 4 & 4 & 16 & 1 & $\begin{array}{l}168 \\
8 \\
7\end{array}$ \\
\hline $\begin{array}{l}\text { Margined White } \\
\text { Mustard White } \\
\text { Cabbage White }\end{array}$ & & 7 & & 3 & 20 & $\begin{array}{l}45 \\
21\end{array}$ & 15 & 1 & 9 & $\begin{array}{l}46 \\
75\end{array}$ \\
\hline
\end{tabular}




\begin{tabular}{|c|c|c|c|c|c|c|c|c|c|c|}
\hline SPECIES NAME & DMP & $\operatorname{Reg}$ & FtQ & LML & Sto & Bjo & Pre & $\mathrm{PaH}$ & $\mathrm{NiF}$ & totals \\
\hline $\begin{array}{l}\text { Large Marble } \\
\text { Olympia Marble } \\
\text { Unident. Sulphur } \\
\text { Clouded Sulphur }\end{array}$ & 1 & 8 & 1 & $\begin{array}{l}10 \\
10\end{array}$ & $\begin{array}{l}29 \\
402\end{array}$ & 405 & 105 & 67 & $\begin{array}{l}2 \\
16\end{array}$ & $\begin{array}{l}41 \\
1015\end{array}$ \\
\hline $\begin{array}{l}\text { Orange Sulphur } \\
\text { Q. Alexandra's Sulphur } \\
\text { Christina Sulphur }\end{array}$ & & & & 2 & $\begin{array}{l}2 \\
3\end{array}$ & & 2 & 1 & 1 & $\begin{array}{l}5 \\
6\end{array}$ \\
\hline $\begin{array}{l}\text { Giant Sulphur } \\
\text { Pink-edged Sulphur } \\
\text { Harvester }\end{array}$ & & & & & & 75 & & 31 & & 106 \\
\hline $\begin{array}{l}\text { Unident. Copper } \\
\text { Gray Copper } \\
\text { Bronze Copper }\end{array}$ & & & & 4 & & & & & & 4 \\
\hline $\begin{array}{l}\text { Ruddy Copper } \\
\text { Dorcas Copper } \\
\text { Purplish Copper }\end{array}$ & & 8 & & 10 & 1 & & & 12 & 3 & $\begin{array}{l}15 \\
19\end{array}$ \\
\hline $\begin{array}{l}\text { Acadian Hairstreak } \\
\text { Coral Hairstreak } \\
\text { Edwards' Hairstreak }\end{array}$ & & & & & & & 1 & & 2 & 3 \\
\hline $\begin{array}{l}\text { Branded Hairstreak } \\
\text { Striped Hairstreak } \\
\text { Unident. Elfin }\end{array}$ & & & & & & & & & & \\
\hline \multicolumn{11}{|l|}{$\begin{array}{l}\text { Brown Elfin } \\
\text { Hoary Elfin } \\
\text { Eastern Pine Elfin }\end{array}$} \\
\hline $\begin{array}{l}\text { Western Pine Elfin } \\
\text { Gray Hairstreak } \\
\text { Unident. Blue }\end{array}$ & & & & 7 & 3 & & 5 & & & 15 \\
\hline $\begin{array}{l}\text { West. Tailed Blue } \\
\text { Spring Azure } \\
\text { Summer Azure }\end{array}$ & $\begin{array}{l}11 \\
5\end{array}$ & 1 & 10 & & 6 & 8 & 3 & 13 & & $\begin{array}{l}52 \\
5\end{array}$ \\
\hline $\begin{array}{l}\text { Rocky Mt. Dotted Blue } \\
\text { Arrowhead Blue } \\
\text { Silvery Blue }\end{array}$ & 21 & 30 & 11 & 3 & 18 & 130 & 1 & 43 & & 257 \\
\hline $\begin{array}{l}\text { Northern Blue } \\
\text { Melissa Blue } \\
\text { Greenish Blue }\end{array}$ & 4 & $\begin{array}{l}6 \\
8\end{array}$ & $\begin{array}{l}3 \\
3\end{array}$ & $\begin{array}{l}7 \\
7\end{array}$ & $\begin{array}{l}3 \\
2\end{array}$ & $\begin{array}{l}10 \\
2\end{array}$ & $\begin{array}{l}1 \\
9\end{array}$ & 7 & & $\begin{array}{l}17 \\
20 \\
35\end{array}$ \\
\hline \multicolumn{11}{|l|}{$\begin{array}{l}\text { Boisduval's Blue } \\
\text { Lupine (Acmon) Blue } \\
\text { Prairie Arctic Blue }\end{array}$} \\
\hline $\begin{array}{l}\text { Unident. large Fritillary } \\
\text { Variegated Fritillary } \\
\text { Great Spangled Frit. }\end{array}$ & & 3 & & 1 & $\begin{array}{l}47 \\
4 \\
11\end{array}$ & $\begin{array}{l}2 \\
2\end{array}$ & $\begin{array}{l}8 \\
8\end{array}$ & 3 & $\begin{array}{l}30 \\
1 \\
15\end{array}$ & $\begin{array}{l}86 \\
10 \\
39\end{array}$ \\
\hline
\end{tabular}




\begin{tabular}{|c|c|c|c|c|c|c|c|c|c|c|}
\hline SPECIES NAME & DMP & Reg & FtQ & LML & Sto & Bjo & Pre & $\mathrm{PaH}$ & NiF & totals \\
\hline \multicolumn{11}{|l|}{$\begin{array}{l}\text { Aphrodite Fritillary } \\
\text { Edwards' Fritillary } \\
\text { Zerene Fritillary }\end{array}$} \\
\hline $\begin{array}{l}\text { Callippe Fritillary } \\
\text { Atlantis Fritillary } \\
\text { Northwestern Fritillary }\end{array}$ & & & $\begin{array}{l}2 \\
11\end{array}$ & & $\begin{array}{l}1 \\
3\end{array}$ & $\begin{array}{l}11 \\
14\end{array}$ & 2 & $\begin{array}{l}256 \\
23\end{array}$ & $\begin{array}{l}1 \\
13\end{array}$ & $\begin{array}{l}3 \\
268 \\
66\end{array}$ \\
\hline $\begin{array}{l}\text { Mormon Fritillary } \\
\text { Unident. small Fritillary } \\
\text { Bog Fritillary }\end{array}$ & & & & & 2 & & & & 23 & 25 \\
\hline $\begin{array}{l}\text { Silver-bordered Fritillary } \\
\text { Meadow Fritillary } \\
\text { Frigga Fritillary }\end{array}$ & & & & 1 & $\begin{array}{l}1 \\
1\end{array}$ & & & 3 & $\begin{array}{l}2 \\
12\end{array}$ & $\begin{array}{l}6 \\
14\end{array}$ \\
\hline $\begin{array}{l}\text { Freija Fritillary } \\
\text { Arctic Fritillary } \\
\text { Gorgone Checkerspot }\end{array}$ & & & & & & & & 2 & 35 & 37 \\
\hline $\begin{array}{l}\text { Sagebrush Checkerspot } \\
\text { Unident. Crescent } \\
\text { Pearl Crescent }\end{array}$ & & 2 & & 3 & $\begin{array}{l}12 \\
4\end{array}$ & & 1 & & 3 & $\begin{array}{l}19 \\
6\end{array}$ \\
\hline $\begin{array}{l}\text { Northern Crescent } \\
\text { Tawny Crescent } \\
\text { Variable Checkerspot }\end{array}$ & 1 & 1 & $\begin{array}{l}9 \\
3\end{array}$ & 7 & $\begin{array}{l}9 \\
1\end{array}$ & $\begin{array}{l}125 \\
7\end{array}$ & 66 & $\begin{array}{l}47 \\
6\end{array}$ & & $\begin{array}{l}265 \\
17\end{array}$ \\
\hline $\begin{array}{l}\text { Unident. Comma } \\
\text { Eastern Comma } \\
\text { Satyr Comma }\end{array}$ & 3 & & & & 3 & 1 & 1 & 3 & $\begin{array}{l}4 \\
2\end{array}$ & $\begin{array}{l}5 \\
12\end{array}$ \\
\hline $\begin{array}{l}\text { Green Comma } \\
\text { Hoary Comma } \\
\text { Gray Comma }\end{array}$ & $\begin{array}{l}2 \\
1\end{array}$ & & & & & & & $\begin{array}{l}18 \\
1\end{array}$ & & $\begin{array}{l}20 \\
2\end{array}$ \\
\hline $\begin{array}{l}\text { Compton Tortoiseshell } \\
\text { Mourning Cloak } \\
\text { Milbert's Tortoiseshell } \\
\text { Painted Lady }\end{array}$ & & $\begin{array}{l}3 \\
3\end{array}$ & 2 & $\begin{array}{l}1 \\
2\end{array}$ & $\begin{array}{l}4 \\
2\end{array}$ & $\begin{array}{l}1 \\
27\end{array}$ & 1 & $\begin{array}{l}4 \\
1 \\
67\end{array}$ & 2 & $\begin{array}{l}4 \\
9 \\
104 \\
3 \\
\end{array}$ \\
\hline $\begin{array}{l}\text { Red Admiral } \\
\text { White Admiral } \\
\text { Viceroy }\end{array}$ & 11 & $\begin{array}{l}11 \\
5\end{array}$ & $\begin{array}{l}10 \\
31\end{array}$ & $\begin{array}{l}5 \\
8 \\
2\end{array}$ & $\begin{array}{l}11 \\
96 \\
1\end{array}$ & $\begin{array}{l}22 \\
52 \\
2\end{array}$ & $\begin{array}{l}1 \\
21\end{array}$ & $\begin{array}{l}1 \\
54 \\
7\end{array}$ & 5 & $\begin{array}{l}72 \\
272 \\
12\end{array}$ \\
\hline $\begin{array}{l}\text { Northern Pearly-Eye } \\
\text { Eyed Brown } \\
\text { Little Wood-Satyr }\end{array}$ & & & & & & 12 & 11 & 3 & & 26 \\
\hline $\begin{array}{l}\text { Common Ringlet } \\
\text { Unident. Wood-Nymph } \\
\text { Com. Wood-Nymph }\end{array}$ & & 110 & 11 & 29 & $\begin{array}{l}10 \\
6\end{array}$ & $\begin{array}{l}3 \\
15\end{array}$ & 60 & 1 & 174 & $\begin{array}{l}164 \\
255 \\
\end{array}$ \\
\hline $\begin{array}{l}\text { Small Wood-Nymph } \\
\text { Unident. Alpine } \\
\text { Taiga Alpine }\end{array}$ & & & & & & & & & & \\
\hline
\end{tabular}




\begin{tabular}{|c|c|c|c|c|c|c|c|c|c|c|}
\hline SPECIES NAME & DMP & $\operatorname{Reg}$ & FtQ & LML & Sto & Bjo & Pre & $\mathrm{PaH}$ & NiF & totals \\
\hline $\begin{array}{l}\text { Red-disked Alpine } \\
\text { Common Alpine } \\
\text { Ridings' Satyr }\end{array}$ & 9 & 1 & & & & & & & & 10 \\
\hline $\begin{array}{l}\text { Unident. Arctic } \\
\text { Macoun's Arctic } \\
\text { Unler's Arctic }\end{array}$ & & 1 & & & & & & 1 & & $\begin{array}{l}1 \\
1\end{array}$ \\
\hline $\begin{array}{l}\text { Jutta Arctic } \\
\text { Monarch } \\
\text { Unident. butterfly }\end{array}$ & & 33 & 13 & 4 & 1 & & 21 & & 1 & 73 \\
\hline Total \# of species & 16 & 24 & 21 & 22 & 36 & 26 & 21 & 33 & 18 & 60 \\
\hline Total \# of butterflies & 201 & 315 & 186 & 175 & 785 & 1039 & 380 & 750 & 359 & 4007 \\
\hline Count Location & DMP & Reg & $\mathrm{FtQ}$ & LML & Sto & Bjo & Pre & $\mathrm{PaH}$ & $\mathrm{NiF}$ & totals \\
\hline
\end{tabular}

${ }^{\star} \mathrm{DMP}=$ Duck Mt. Prov. Park, Reg = Regina, FtQ = Fort Qu'Appelle, LML $=$ Last Mt. Lake National Wildlife Area, Sto $=$ Saskatoon, Bjo = Bjorkdale, Pre $=$ Preeceville, $\mathrm{PaH}=$ Pasqua Hills, NiF $=$ Nisbet Forest

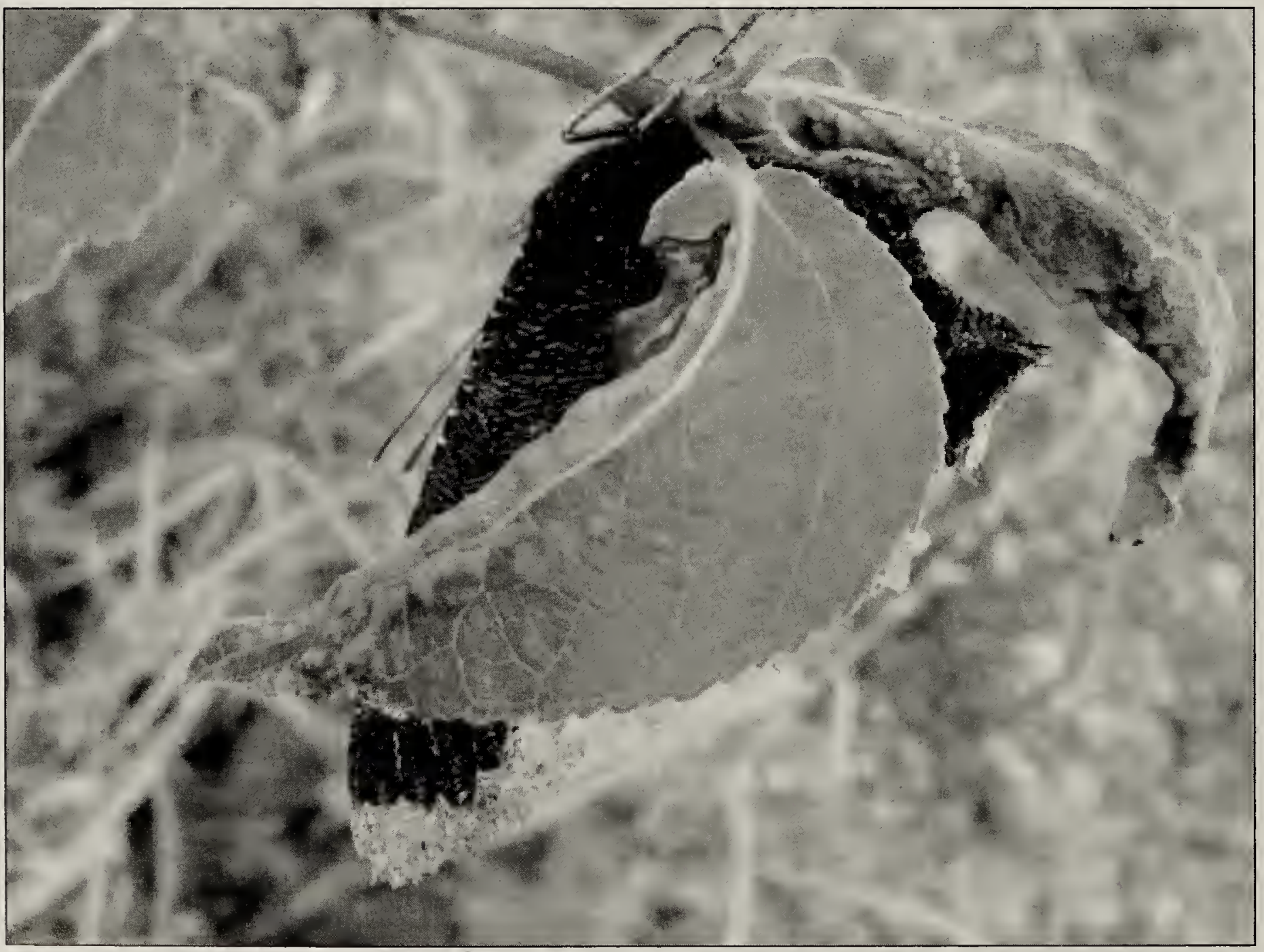

Figure 1. Mourning Cloak butterfly laying eggs on a Balsam Poplar, 18 July 2007, Pasquia Hills

John Kozial 
Mountain, 45 Mustard Whites at Bjorkdale, 75 Pink-edged Sulphurs at Bjorkdale, 12 Dorcas Coppers at Pasquia Hills, 130 Silvery Blues at Bjorkdale, 256 Atlantis Fritillary at Pasquia Hills, 125 Northern Crescents at Bjorkdale, 18 Green Commas at Pasquia Hills, 67 Milbert's Tortoiseshells at Pasquia Hills, 96 White Admirals at Saskatoon, and 33 Monarchs at Regina and 21 at Preeceville.

As in 2006, virtually all of the common species that might be expected considering the dates and locations of the nine counts were found in 2007. The most conspicuously absent species was Aphrodite Fritillary. The more common transient species also were all represented in at least one count: Common Checkered Skipper, present on two counts (total 2 individuals); Variegated Fritillary on 4 counts (total 10); Painted Lady on one count (total 3 ); Red Admiral on 8 counts (total 72); and Monarch on 6 counts (total 73). The only new species for the Saskatchewan 1JC counts was a Kahli Swallowtail at Last Mountain Lake.

Table 2 shows only the adult species that were seen on the counts.
Both Monarchs and Mourning Cloaks were found in immature stages as well. One Monarch larva was found feeding on milkweed on the Nisbet Forest count. No adults were seen on that count. On the Pasquia Hills count, John Kozial observed a worn, adult Mourning Cloak depositing about a dozen eggs in a partial ring on a small Balsam Poplar (Populus balsamifera) sapling at 1:10 p.m.(Figure 1). He also came across a total of 122 larvae on four small trees (all $120 \mathrm{~cm}$ tall or less): 29 larvae were on two Aspen Poplars (Populus tremuloides) (Figure 2), 85 were on a Pussy Willow (Salix discolor) and 8 on a Balsam Poplar.

\section{Acknowledgements}

We'd like to thank the count participants, and the count leaders and compilers (Dallas Fairburn, Ron Hooper, John Kozial, Ray Poulin and Michael Williams), as well as Ron Hooper and Felix Sperling for assistance in identifying the Kahli Swallowtail.

1. GOLLOP, M. and A. LEIGHTON. 2005. Results of the 2005 International Butterfly Counts in Saskatchewan. Blue Jay 63:193-199

2. GOLLOP, M. and A. LEIGHTON. 2007. Results of the 2006 International Butterfly Counts in Saskatchewan. Blue Jay 65:89-95

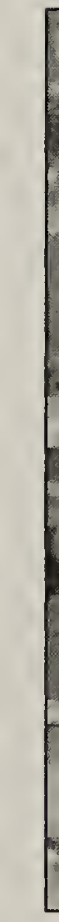

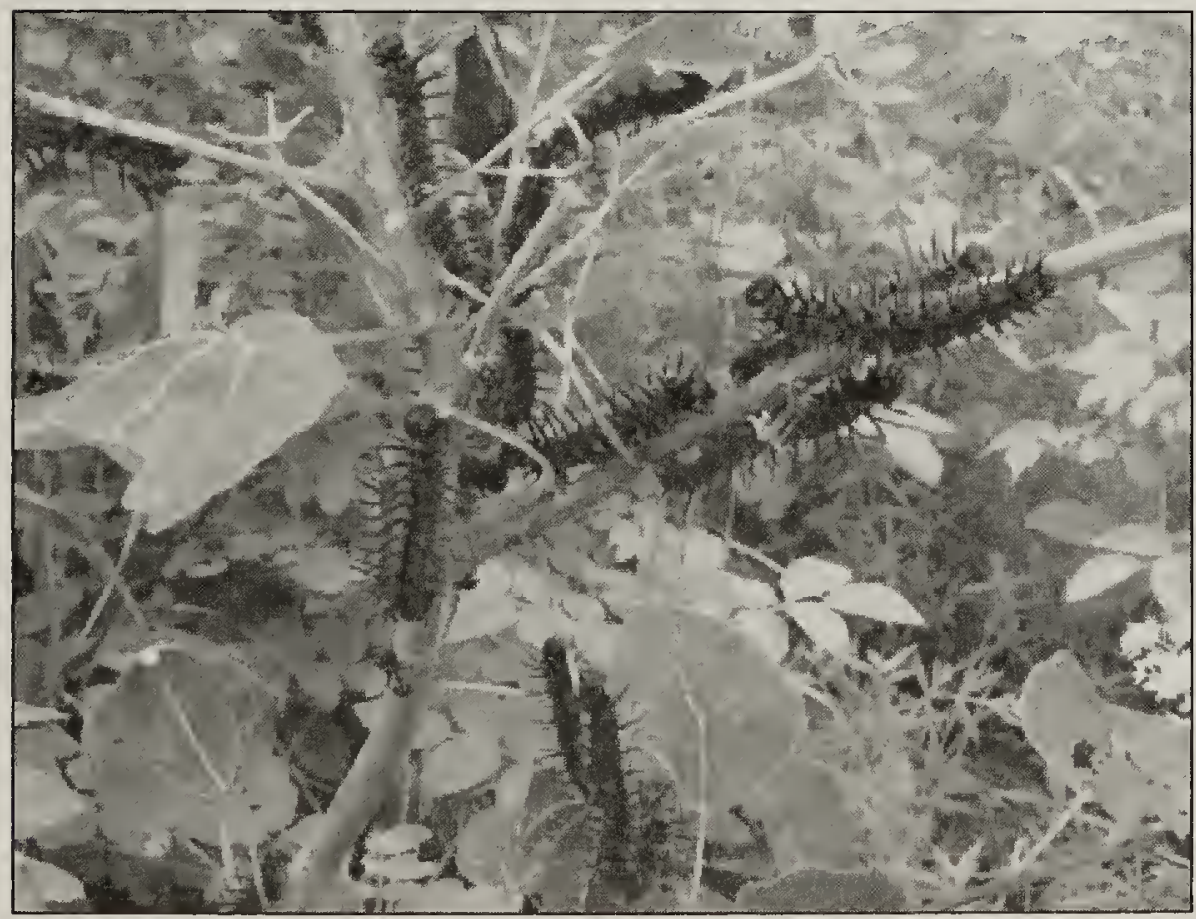

Figure 2. Mourning Cloak larvae on an Aspen Poplar on 18 July 2007, Pasquia Hills John Kozial 